\title{
A case of multiple pulmonary nodules diagnosed as pulmonary melanoma without extra-pulmonary site
}

\author{
Yoshihiro Miyashita* ${ }^{1}$, Miyuki Kobayashi ${ }^{1}$, Yumiko Kakizaki ${ }^{1}$, Toshiharu Tsutsui ${ }^{1}$, Takashi Kumagai ${ }^{1}$, Hiroaki \\ Kobayashi ${ }^{1}$, Haruna Yamaki ${ }^{1}$, Chisa Ohmori ${ }^{1}$, Toshio Oyama ${ }^{2}$ \\ ${ }^{1}$ Lung Cancer and Respiratory Disease Center, Yamanashi Central Hospital, 1-1-1 Fujimi, Kofu, Yamanashi, Japan \\ ${ }^{2}$ Department of Pathology, Yamanashi Central Hospital, 1-1-1 Fujimi, Kofu, Yamanashi, Japan
}

Received: December 28, 2020

Accepted: January 18, 2021

Online Published: January 26, 2021

DOI: $10.5430 /$ crim.v8n1p1

URL: https://doi.org/10.5430/crim.v8n1p1

\begin{abstract}
A 67-year-old patient visited the hospital for the evaluation of pulmonary nodular lesions detected in his chest radiograph during an annual screening. He was asymptomatic and transbronchial tumor biopsy of the nodule in the right upper lobe revealed features of pulmonary sarcoma, which did not resemble any of the histolopathological categories of primary lung cancer. A positive immunological staining for both HMB-45 and Melan-A resulted in the final diagnosis of melanoma. It is important for a respiratory clinician to remind the possibility of a melanoma, when tumors cannot be histopathologically classified under any of the categories of primary lung cancer.
\end{abstract}

Key Words: Malignant melanoma, Pulmonary nodule, Metastatic

\section{INTRODUCTION}

Primary melanoma of the lung is uncommon, accounting for just $0.01 \%$ of all lung tumors. ${ }^{[1]}$ Pulmonary melanoma without evidence of extra-pulmonary lesions (primary pulmonary melanoma) is very rare and its clinical significance has not yet been fully understood. ${ }^{[2-4]}$ This could either be a true primary pulmonary melanoma or metastatic disease where the primary has been missed or has regressed. We report a case of pulmonary melanoma where the patient presented with two nodules in the right lung with no evidence of extrapulmonary disease, including skin and ocular lesions. We could make a correct diagnosis of melanoma by immunohistochemical staining considering for an unusual histological diagnosis of lung tumor.

\section{Case presentation}

A 67-year-old patient visited our hospital in February 2018, for the evaluation of pulmonary nodular lesions that had been detected in his chest radiograph during an annual screening (see Figure 1a). He had a past smoking history of ten packyears and a history of several years with asbestos exposure in his twenties. He was asymptomatic and his physical examination was normal. There were no skin and ocular lesions observed. Two nodular lesions were seen on chest CT, one each in the upper and middle lobes of the right lung(see Figure 1b, 1c). In addition, FDG (fluoro-deoxyglucose) accumulation was observed in both the nodules (see Figure 2). There were no other lesions observed in the CT. The nodules were suspected to be malignant, possibly metastatic, and bronchoscopy with ultrasound-guided sheath was performed. Transbronchial tumor biopsy of the nodule in the right up-

*Correspondence: Yoshihiro Miyashita; Email: y-miyashita@ych.pref.yamanashi.jp; Address: Lung Cancer and Respiratory Disease CenterYamanashi Central Hospital 1-1-1 Fujimi, Kofu, Yamanashi, Japan. 
per lobe revealed the findings of pulmonary sarcoma, and could not be categorized histopathologically under primary lung cancers (see Figure 3 a,b). Immunohistochemical staining was done and a positive staining for both HMB-45 and Melan-A resulted in the final diagnosis of melanoma (see Figure $3 \mathrm{c}, \mathrm{d}$ ). Systemic evaluation, including PET (positron emission tomography) imaging, was done to look for malignancies in the gastrointestinal tract, bone, skin, and head and neck regions. However, a primary lesion could not be found. Clinical laboratory tests including tumor markers were nor- mal. Genomic analysis of the obtained tissue was positive for BRAF mutation (V600E). Consequently, molecular targeted therapy for melanoma with dabrafenib and trametinib was started from April 2018. There was shrinking of the nodular lesions soon after the induction of therapy (see Figure 4a). CT and PET evaluation in June, 2018 did not detect any residual lesions and the treatment was completed without any serious adverse events (see Figure 4 b,c). The patient has been on follow-up till date (July 2020) and no recurrence has been observed.

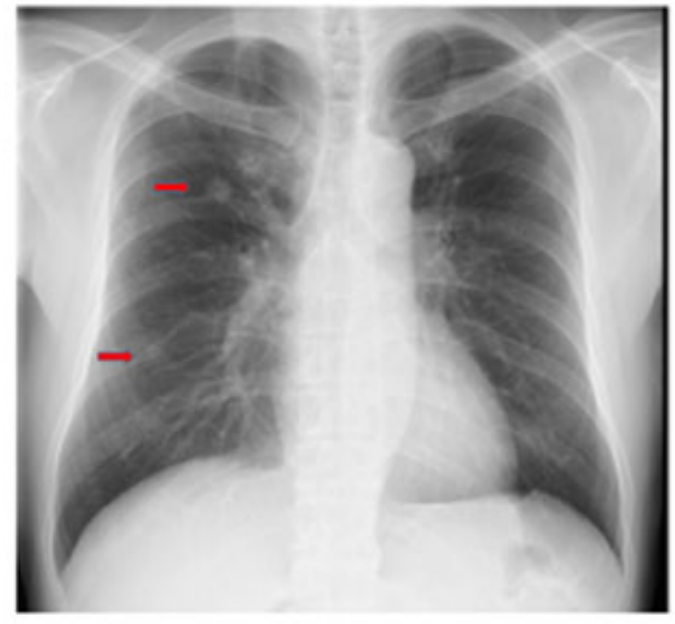

(a)

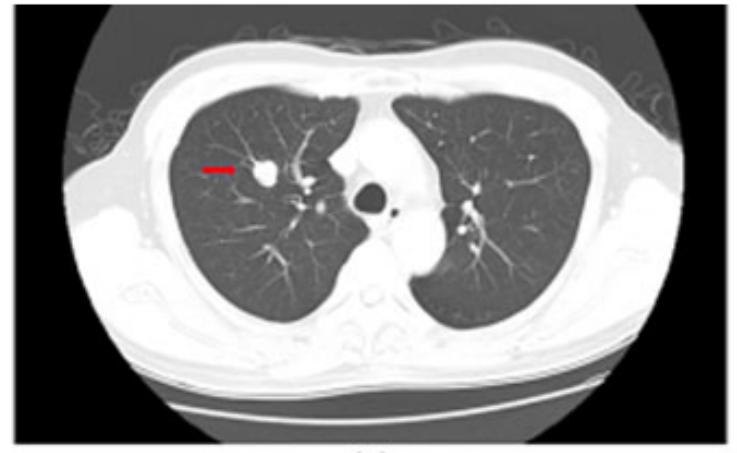

(b)

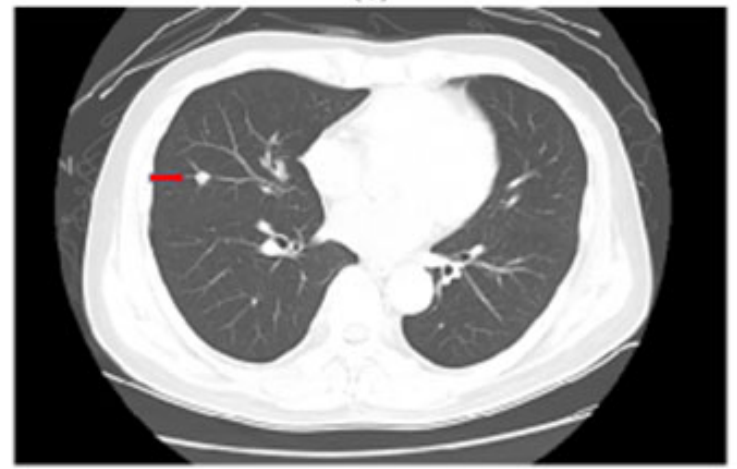

(c)

Figure 1. Radiological findings a: Two nodular lesions (red arrow) seen in the chest radiograph. b,c: The nodular lesions seen on the chest CT (red arrow), in the upper lobe (b) and the middle lobe (c) of the right lung.

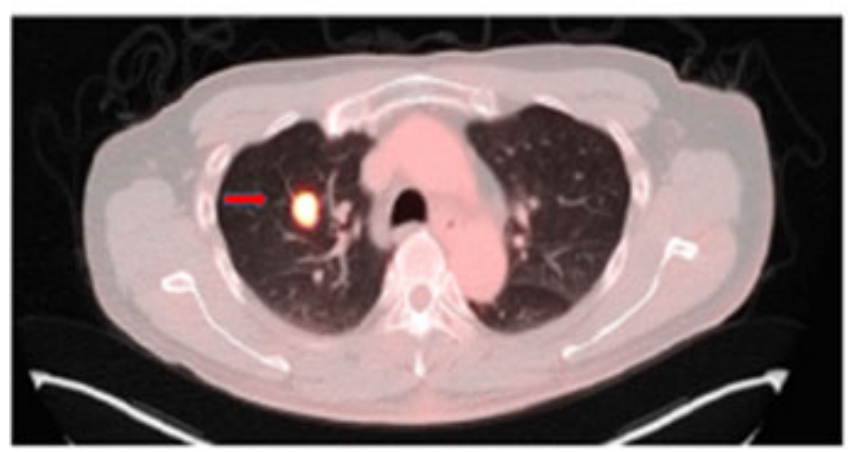

(a)

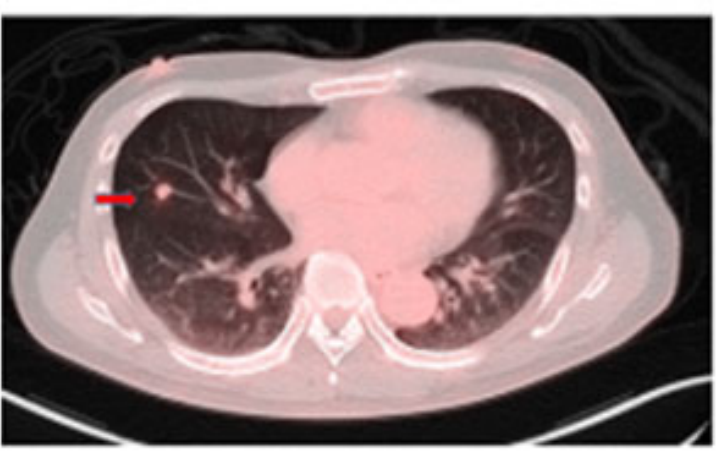

(b)

Figure 2. FDG accumulation was observed in both the nodules of right upper (a), and middle (b) lobe. 

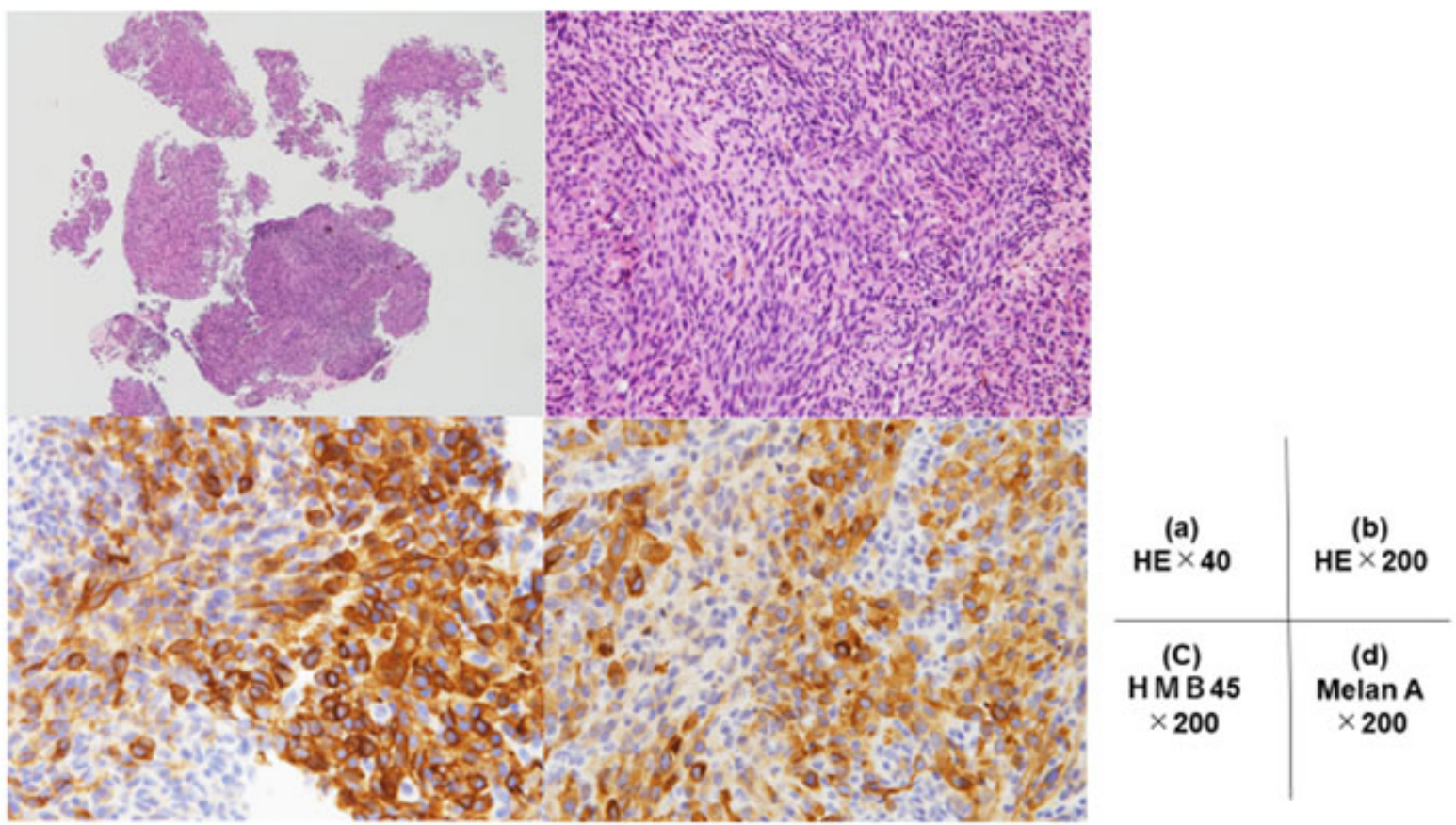

Figure 3. Histopathological findings from the biopsy of the lesion in the right upper lobe. a,b: Features suggestive of pulmonary sarcoma with spindle cells. c,d: Immunohistochemical staining for HMB45 and Melan A for melanoma were positive.

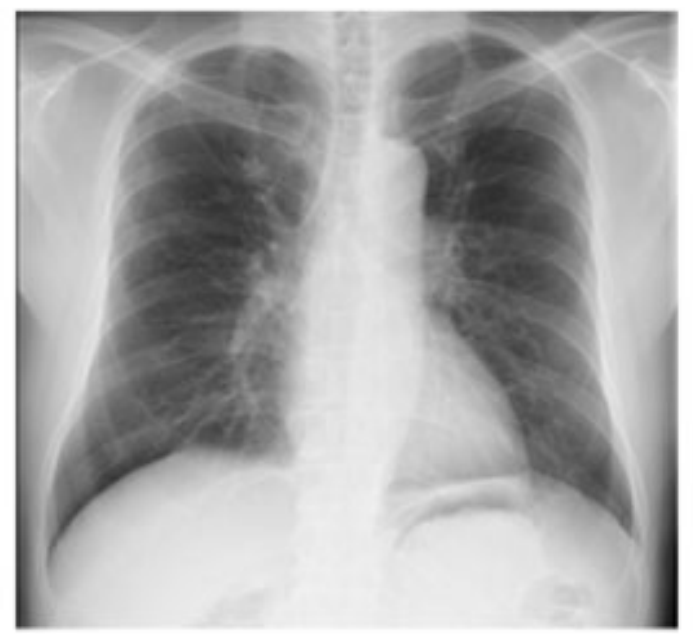

(a)

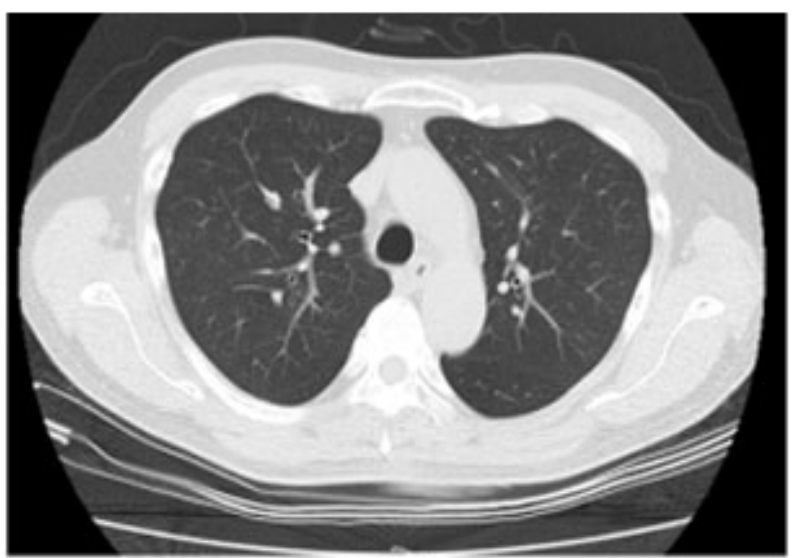

(b)

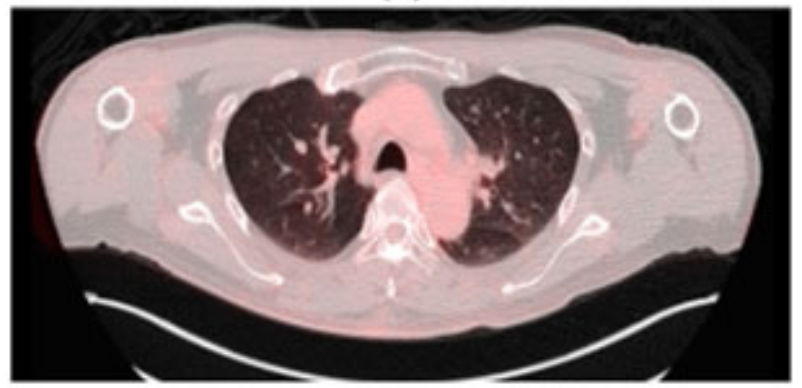

(c)

Figure 4. Post-treatment imaging. a: Normal chest radiograph. b, c: CT and PET scan images showing no evidence of residual lesions. 


\section{Discussion}

A retrospective study concerning about 103 patients of metastatic melanoma with undetermined primary sites reported the difficulty of disclosing a primary site (5). Only one patient with choroidal melanoma was disclosed as the possible primary site pointed out by ophthalmologist in that report. ${ }^{[5]}$ In this case, we considered the possibility of either a primary lung cancer or metastatic malignant pulmonary nodules. There were no extra-pulmonary lesions, the histopathological findings were inconclusive, and the CT findings suggested the possibility of metastatic pulmonary lesions. Thus, immunohistochemical staining was vital to arrive at the diagnosis of melanoma. Considering the recommended criteria ${ }^{[1]}$ of primary pulmonary melanomas, the pulmonary nodule should be a solitary lesion. Metastatic melanoma in the lung presents as a solitary lesion in only $1 \%$ of the cases. ${ }^{[2]}$ Cutaneous melanomas are known to sometimes exhibit spontaneous regression after metastasis, which makes it difficult to differentiate between a primary malignant melanoma in a non-cutaneous site and a metastatic lesion. ${ }^{[2]}$ Therefore the presence of multiple nodular lesions as seen in this case would more likely suggest a metastatic melanoma in spite of an undetermined primary site. Cancer immunotherapy has seen lot of developments in recent years. The monoclonal anti-PD-1 antibodies nivolumab and pembrolizumab were approved by the Food and Drug Administration (FDA) in 2014 for treating metastatic cutaneous melanoma. ${ }^{[6]}$ The discovery of BRAF mutation in almost half of all melanoma patients has led to a dramatic change in the treatment of melanoma. Molecular targeted therapy with dabrafenib and trametinib is currently used depending on the presence of BRAF mutation. In this case, we have observed a disease free survival time of two years after the administration of the treatment, without any adverse events.

\section{Conclusion}

This case shows that, a respiratory clinician evaluating suspicious pulmonary nodules should be aware of the possibility of melanoma when, after biopsy, the lesion cannot be histopathologically categorized under primary lung cancers.

\section{CONFlicts of InTERest Disclosure}

The authors have declared no conflicts of interest.

\section{REFERENCES}

[1] Dountsis A, Zisis C, Karagianni E, et al. Primary Malignant Melanoma of the Lung: A Case Report. World J Surg Oncol. 2003 1(1): 26. PMid:14750982. https://doi.org/10.1186/1477-7 819-1-26

[2] Shi Y, Bing Z, Xu X, et al. Primary pulmonary malignant melanoma: Case report and literature review. Thorac Cancer. 2018; 9(9): 11851189. PMid:30062692. https://doi .org/10.1111/1759-7714. 12798

[3] Kyriakopoulos C, Zarkavelis G, Andrianopoulou A, et al. Primary Pulmonary Malignant Melanoma: Report of an Important Entity and Literature Review. Case Rep Oncol Med. 2017; 2017: 8654326. PMid:28352484. https://doi .org/10.1155/2017/8654326
[4] Seitelman E, Donenfeld P, Kay K, et al. Successful treatment of primary pulmonary melanoma. J Thorac Dis. 2011; 3(3): 207-208.

[5] Tina T, Helle K, Krzysztof TD. Extensive screening for primary tumor is redundant in melanoma of unknown primary. J Surg Oncol 2011; 104(7): 724-727. PMid:21721009. https://doi .org/10.1 002/jso. 21994

[6] Yun S, Vincelette ND, Green MR, et al. Targeting immune checkpoints in unresectable metastatic cutaneous melanoma: a systematic review and meta-analysis of anti-CTLA-4 and anti-PD-1 agents trials. Cancer Med. 2016; 5(7): 1481-1491. PMid:27167347. https : //doi.org/10.1002/cam4.732 